УДК 517.983

\title{
ЛИНЕЙНЫЕ КРАЕВЫЕ ЗАДАЧИ ДЛЯ НОРМАЛЬНО РАЗРЕШИМЫХ ОПЕРАТОРНЫХ УРАВНЕНИЙ В БАНАХОВОМ ПРОСТРАНСТВЕ
}

\author{
(c) 2014 г. А. М. Самойленко, А. А. Бойчук, В. Ф. Журавлев
}

\begin{abstract}
Рассмотрены линейные краевые задачи для операторных уравнений с обобщенно обратимым оператором в банаховом или гильбертовом пространстве. Получены условия разрешимости таких задач и указана структура их решений. Построен обобщенный оператор Грина, изучены его свойства и связь с обобщенно-обратным оператором линейной краевой задачи. Предложенный подход детально проиллюстрирован на примере.
\end{abstract}

DOI: $10.1134 /$ S0374064114030054

Классическая теория периодических задач для различных классов дифференциальных уравнений [1-3] начала свое развитие еще до появления методов функционального анализа. Дальнейшее ее развитие стало возможным [4-8] с применением функционального анализа в качестве аппарата для исследования общих краевых задач. Так, с применением теории обобщенного обращения матриц [9] методы исследования периодических краевых задач были обобщены на общие краевые задачи для различных классов функционально-дифференциальных уравнений: обыкновенных дифференциальных систем в критических (резонансных) случаях [10]; дифференциальных систем с запаздывающим аргументом [11]; обыкновенных дифференциальных систем с импульсным воздействием $[12,13]$. Доказаны общие теоремы о разрешимости и представлении решений критических краевых задач для различных классов линейных и нелинейных уравнений, обобщены пространства, в которых рассматривались эти краевые задачи.

Дальнейшим обобщением стала теория краевых задач для обыкновенных дифференциальных уравнений в банаховом пространстве, когда конечномерное евклидово пространство значений искомой функции заменялось на общее банахово пространство [14]. Так, в работе [15] получен критерий существования решений линейных краевых задач в критическом случае для обыкновенных дифференциальных уравнений в банаховом пространстве. В монографии $[16$, с. 266] исследовались условия существования периодических решений дифференциальных и разностных уравнений в банаховом пространстве $\mathbf{m}$ ограниченных числовых последовательностей. В работе [17] получены условия существования и бифуркации ограниченных на всей оси $\mathbb{R}=(-\infty,+\infty)$ решений слабовозмущенного дифференциального уравнения в банаховом пространстве.

С точки зрения теории операторов в функциональных пространствах перечисленные выше краевые задачи обладают следующими особенностями: исходные уравнения этих краевых задач имеют решения при любой правой части. Согласно терминологии С.Г. Крейна [18, с. 8], такие задачи являются везде разрешимыми. Однако существуют краевые задачи, у которых исходное операторное уравнение не является везде разрешимым, например, задачи для интегро-дифференциальных уравнений [19], задачи для сингулярных дифференциальных систем [20-22]. В связи с этим актуальной остается задача рассмотрения общих краевых задач для не везде разрешимых операторных уравнений в банаховых пространствах, чему и посвящена настоящая работа.

Постановка задачи. Пусть $\mathbf{l}_{\infty}\left(\mathcal{I}, \mathbf{B}_{1}\right)$ - банахово пространство ограниченных векторфункций $z(t)$, определенных на конечном промежутке $\mathcal{I}$ со значениями в некотором банаховом пространстве $\mathbf{B}_{1}, \quad z(\cdot): \mathcal{I} \rightarrow \mathbf{B}_{1}$ с нормой \|\|$z\left\|=\sup _{t \in \mathcal{I}}\right\| z(t) \|_{\mathbf{B}_{1}}$, a $\mathbf{l}_{\infty}\left(\mathcal{I}, \mathbf{B}_{2}\right)$ - банахово пространство ограниченных вектор-функций $\varphi(t)$, определенных на том же промежутке $\mathcal{I}$ 
со значениями в некотором банаховом пространстве $\mathbf{B}_{2}$ с нормой \|\|$\varphi\|\|=\sup _{t \in \mathcal{I}}\|\varphi(t)\|_{\mathbf{B}_{2}}, \quad \mathbf{B}-$ банахово пространство векторов с постоянными компонентами. Обозначения функциональных пространств заимствованы из монографии [23].

Пусть $L: \mathbf{l}_{\infty}\left(\mathcal{I}, \mathbf{B}_{1}\right) \rightarrow \mathbf{l}_{\infty}\left(\mathcal{I}, \mathbf{B}_{2}\right)$ - линейный ограниченный обобщенно-обратимый опеpaтор, $\ell=\operatorname{col}\left(l_{1}, l_{2}, l_{3}, \ldots\right): \mathbf{l}_{\infty}\left(\mathcal{I}, \mathbf{B}_{1}\right) \rightarrow \mathbf{B}$ - линейный ограниченный вектор-функционал. Обобщенная обратимость оператора $L$ означает [24, с. 139], что он нормально разрешим, его нуль-пространство $N(L)$ и ядро $R(L)$ дополняемы в банаховых пространствах $\mathbf{l}_{\infty}\left(\mathcal{I}, \mathbf{B}_{1}\right)$ и $\mathbf{l}_{\infty}\left(\mathcal{I}, \mathbf{B}_{2}\right)$ соответственно. При этом существуют [25] ограниченные проекторы $\mathcal{P}_{N(L)}:$ $\mathbf{l}_{\infty}\left(\mathcal{I}, \mathbf{B}_{1}\right) \rightarrow N(L)$ и $\mathcal{P}_{Y_{L}}: \mathbf{l}_{\infty}\left(\mathcal{I}, \mathbf{B}_{2}\right) \rightarrow Y_{L}$, которые разбивают пространства $\mathbf{l}_{\infty}\left(\mathcal{I}, \mathbf{B}_{1}\right)$ и $\mathbf{l}_{\infty}\left(\mathcal{I}, \mathbf{B}_{2}\right)$ в прямые суммы замкнутых подпространств

$$
\mathbf{l}_{\infty}\left(\mathcal{I}, \mathbf{B}_{1}\right)=N(L) \oplus X_{L}, \quad \mathbf{l}_{\infty}\left(\mathcal{I}, \mathbf{B}_{2}\right)=Y_{L} \oplus R(L) .
$$

Традиционно обобщенно обратимые операторы включают в себя следующие операторы: фредгольмовы $\left(\operatorname{dim} N(L)=\operatorname{dim} Y_{L}<\infty\right)[26] ;$ нётеровы $\left(\operatorname{dim} N(L)=n<\infty, \operatorname{dim} Y_{L}=d<\infty\right.$, $n \neq d)$ [27], где теория Фредгольма обобщена на сингулярные интегральные уравнения; $n$-нормальные $\left(\operatorname{dim} N(L)=n<\infty, \operatorname{dim} Y_{L}=\infty\right)$ с дополняемым образом; $d$-нормальные $\left(\operatorname{dim} N(L)=\infty, \operatorname{dim} Y_{L}=d<\infty\right)$ с дополняемым ядром [18, с. 27, 31]; топологически фредгольмовы $\left(\operatorname{dim} N(L)=\infty, \quad \operatorname{dim} Y_{L}=\infty, N(L)\right.$ изоморфно $\left.Y_{L}\right)$ (приводимо-обратимые, если оператор действует из банахова пространства в себя [28, с. 24]); топологически нётеровы $\left(\operatorname{dim} N(L)=\infty, \operatorname{dim} Y_{L}=\infty\right)[29]$

Подчеркнем, что в русскоязычной литературе различают фредгольмовы и нётеровы операторы $[11,13,18,23]$, а в англоязычной оба эти класса называют фредгольмовыми. В иностранной литературе $n$-, $d$-нормальные операторы называют полуфредгольмовыми или полунётеровыми, среди которых различают $\Phi_{+}$-операторы $(\operatorname{dim} N(L)<\infty)$ и $\Phi_{-}$-операторы $\left(\operatorname{dim} Y_{L}<\infty\right)$ [30].

В данной работе будут получены условия разрешимости общих краевых задач для операторных уравнений с обобщенно-обратимыми операторами в абстрактных банаховых пространствах и изучены свойства обобщенного оператора Грина таких краевых задач.

Краевая задача. Рассмотрим задачу о необходимых и достаточных условиях разрешимости и о структуре множества решений $z(t) \in \mathbf{l}_{\infty}\left(\mathcal{I}, \mathbf{B}_{1}\right)$ линейной краевой задачи

$$
\begin{gathered}
(L z)(t)=\varphi(t), \\
\ell z(\cdot)=\alpha .
\end{gathered}
$$

Нормально разрешимое уравнение (1) разрешимо для тех и только тех $\varphi(t) \in \mathbf{l}_{\infty}\left(\mathcal{I}, \mathbf{B}_{2}\right)$, которые удовлетворяют условию [18, с. 14; 24, с. 133]

$$
\left(\mathcal{P}_{Y_{L}} \varphi\right)(t)=0
$$

где $\mathcal{P}_{Y_{L}}: \mathbf{l}_{\infty}\left(\mathcal{I}, \mathbf{B}_{2}\right) \rightarrow Y_{L} \subset \mathbf{l}_{\infty}\left(\mathcal{I}, \mathbf{B}_{2}\right)$ - проектор на подпространство $Y_{L}$, изоморфное нульпространству $N\left(L^{*}\right)$ оператора $L^{*}$, сопряженного к оператору $L$.

При выполнении условия (3) общее решение уравнения (1) представимо в виде

$$
z(t)=\left(\mathcal{P}_{N(L)} \hat{z}\right)(t)+\left(L^{-} \varphi\right)(t)
$$

где $\hat{z}(t)$ - произвольный элемент пространства $\mathbf{l}_{\infty}\left(\mathcal{I}, \mathbf{B}_{1}\right), \quad L^{-}$- ограниченный обобщеннообратный оператор к оператору $L[11$, с. $53 ; 13$, с. 40; 31].

Для того чтобы решение (4) неоднородного операторного уравнения (1) было решением краевой задачи $(1),(2)$, необходимо и достаточно, чтобы элемент $\hat{z}(t)=\hat{z}_{0}(t) \in \mathbf{l}_{\infty}\left(\mathcal{I}, \mathbf{B}_{1}\right)$ удовлетворял операторному уравнению

$$
\ell\left(\mathcal{P}_{N(L)} \hat{z}_{0}\right)(\cdot)+\ell\left(L^{-} \varphi\right)(\cdot)=\alpha
$$

полученному после подстановки решения (4) в краевое условие (2). 
Обозначим через $\mathcal{L} *=\ell \mathcal{P}_{N(L)} *$ линейный оператор, действующий из банахова пространства $\mathbf{l}_{\infty}\left(\mathcal{I}, \mathbf{B}_{1}\right)$ в банахово пространство $\mathbf{B}$. Оператор $\mathcal{L}$ является ограниченным как суперпозиция ограниченных функционала $\ell$ и проектора $\mathcal{P}_{N(L)}$.

Пусть оператор $\mathcal{L}$ принадлежит классу обобщенно-обратимых операторов, т.е. ядро $N(\mathcal{L})$ и образ $R(\mathcal{L})$ оператора $\mathcal{L}$ дополняемы в банаховых пространствах $\mathbf{l}_{\infty}\left(\mathcal{I}, \mathbf{B}_{1}\right)$ и $\mathbf{B}$ соответственно.

Обозначим через $\mathcal{P}_{N(\mathcal{L})}: \mathbf{l}_{\infty}\left(\mathcal{I}, \mathbf{B}_{1}\right) \rightarrow N(\mathcal{L})$ ограниченный проектор банахова пространства $\mathbf{l}_{\infty}\left(\mathcal{I}, \mathbf{B}_{1}\right)$ на нуль-пространство оператора $\mathcal{L}$, через $\mathcal{P}_{Y_{\mathcal{L}}}: \mathbf{B} \rightarrow Y_{\mathcal{L}}$ ограниченный проектор банахова пространства $\mathbf{B}$ на подпространство $Y_{\mathcal{L}} \subset \mathbf{B}$, а через $\mathcal{L}^{-}$линейный ограниченный обобщенно-обратный оператор к оператору $\mathcal{L}$.

Из операторного уравнения

$$
\left(\mathcal{L} \hat{z}_{0}\right)(\cdot)=\alpha-\ell\left(L^{-} \varphi\right)(\cdot)
$$

определим элемент $\hat{z}_{0}(t) \in \mathbf{l}_{\infty}\left(\mathcal{I}, \mathbf{B}_{1}\right)$, при котором решение (4) уравнения $(1)$, существующее при выполнении условия (3), будет решением краевой задачи (1), (2). Поскольку, согласно предположению, оператор $\mathcal{L}$ обобщенно-обратим, а следовательно, нормально разрешим, то уравнение (5) разрешимо тогда и только тогда, когда выполнено условие

$$
\mathcal{P}_{Y_{\mathcal{L}}}\left\{\alpha-\ell\left(L^{-} \varphi\right)(\cdot)\right\}=0 .
$$

При выполнении этого условия уравнение (5) имеет семейство решений вида

$$
\hat{z}_{0}(t)=\left(\mathcal{P}_{N(\mathcal{L})} \hat{z}\right)(t)+\left(\mathcal{L}^{-}\left\{\alpha-\ell\left(L^{-} \varphi\right)(\cdot)\right\}\right)(t),
$$

где $\hat{z}(t)$ - произвольный элемент банахова пространства $\mathbf{l}_{\infty}\left(\mathcal{I}, \mathbf{B}_{1}\right)$.

Подставляя $\hat{z}_{0}(t)$ из $(7)$ вместо $\hat{z}(t)$ в $(4)$, получаем общее решение краевой задачи $(1),(2)$

$$
\begin{gathered}
z(t)=\left(\mathcal{P}_{N(L)}\left\{\left(\mathcal{P}_{N(\mathcal{L})} \hat{z}\right)(\cdot)+\mathcal{L}^{-}\left[\alpha-\ell\left(L^{-} \varphi\right)(\cdot)\right]\right\}\right)(t)+\left(L^{-} \varphi\right)(t)= \\
=\left(\mathcal{P}_{N(L)} \mathcal{P}_{N(\mathcal{L})} \hat{z}\right)(t)+(G \varphi)(t)+\left(\mathcal{P}_{N(L)} \mathcal{L}^{-} \alpha\right)(t) .
\end{gathered}
$$

Здесь $\mathcal{P}_{N(L)} \mathcal{P}_{N(\mathcal{L})}$ - разрешающий оператор [14, с. 147] соответствующей $(1),(2)$ однородной краевой задачи, а оператор $G: \mathbf{l}_{\infty}\left(\mathcal{I}, \mathbf{B}_{2}\right) \rightarrow N(\ell) \subset \mathbf{l}_{\infty}\left(\mathcal{I}, \mathbf{B}_{1}\right)$ вычисляется по формуле

$$
(G \varphi)(t)=\left(L^{-} \varphi\right)(t)-\left(\mathcal{P}_{N(L)} \mathcal{L}^{-} \ell\left(L^{-} \varphi\right)(\cdot)\right)(t)
$$

и называется обобщенным оператором Грина полуоднородной $(\alpha=0)$ краевой задачи $(1),(2)$.

Таким образом, справедлива

Теорема 1. Пусть $L: \mathbf{l}_{\infty}\left(\mathcal{I}, \mathbf{B}_{1}\right) \rightarrow \mathbf{l}_{\infty}\left(\mathcal{I}, \mathbf{B}_{2}\right)$ и $\mathcal{L}: \mathbf{l}_{\infty}\left(\mathcal{I}, \mathbf{B}_{1}\right) \rightarrow \mathbf{B}$ - обобщеннообратимые операторы. Тогда соответствующая (1), (2) однородная краевая задача $(\varphi(t)=0$, $\alpha=0)$ имеет семейство линейно-независимых решений

$$
z(t)=\left(\mathcal{P}_{N(L)} \mathcal{P}_{N(\mathcal{L})} \hat{z}\right)(t)
$$

где $\hat{z}(t)$ - произвольный элемент банахова пространства $\mathbf{l}_{\infty}\left(\mathcal{I}, \mathbf{B}_{1}\right)$.

Неоднородная краевая задача (1), (2) разрешима для тех и только тех $\varphi(t) \in \mathbf{l}_{\infty}\left(\mathcal{I}, \mathbf{B}_{2}\right)$ и $\alpha \in \mathbf{B}$, которые удовлетворяют условиям (3), (6) и при этом имеет семейство решений вида (8):

$$
z(t)=\left(\mathcal{P}_{N(L)} \mathcal{P}_{N(\mathcal{L})} \hat{z}\right)(t)+(G \varphi)(t)+\left(\mathcal{P}_{N(L)} \mathcal{L}^{-} \alpha\right)(t)
$$

где $\mathcal{P}_{N(L)} \mathcal{P}_{N(\mathcal{L})}$ - разрешающий оператор соответствующей (1), (2) однородной краевой задачи, $G$ - обобщенный оператор Грина (9).

Следствие 1. Если операторь $L$ и $\mathcal{L}$ нормально разрешимые и действуют в гильбертовых пространствах, то в теореме 1 вместо обобщенно-обратных операторов $L^{-}$и $\mathcal{L}^{-}$ будут псевдообратные операторы $L^{+}$и $\mathcal{L}^{+}[13]$, а вместо проекторов $\mathcal{P}_{N(L)}, \mathcal{P}_{Y_{L}} u \mathcal{P}_{N(\mathcal{L})}$, 
$\mathcal{P}_{Y_{\mathcal{L}}}$ - ортопроекторы $P_{N(L)}, P_{N\left(L^{*}\right)}$ u $P_{N(\mathcal{L})}, P_{N\left(\mathcal{L}^{*}\right)}$ соответственно. В этом случае формула (10) примет вид

$$
z(t)=\left(P_{N(L)} P_{N(\mathcal{L})} \hat{z}\right)(t)+(G \varphi)(t)+\left(P_{N(L)} \mathcal{L}^{+} \alpha\right)(t)
$$

где $(G \varphi)(t)=\left(L^{+} \varphi\right)(t)-\left(P_{N(L)} \mathcal{L}^{+} \ell\left(L^{+} \varphi\right)(\cdot)\right)(t)$ - обобщенный оператор Грина.

Замечание 1. При рассмотрении линейных краевых задач вида (1), (2) для всюду разрешимого дифференциального оператора $L z(t)=z^{\prime}(t)-A(t) z(t)$, который действует из банахового пространства $\mathbf{C}^{1}(\mathcal{I}, \mathbf{B})$ непрерывно дифференцируемых функций со значениями в банаховом пространстве $\mathbf{B}$ в банахово пространство $\mathbf{C}(\mathcal{I}, \mathbf{B})$ непрерывных вектор-функций с супремум нормой, приходим к известным ранее результатам [15].

Замечание 2. Рассматривая периодические краевые задачи для линейных разностных уравнений в банаховом пространстве $\mathbf{m}$, приходим к известным ранее результатам, полученным в [16] в нерезонансном случае.

Как следствие из теоремы 1 рассмотрим начальную задачу

$$
\begin{gathered}
(L z)(t)=\varphi(t), \\
\ell z(\cdot) \equiv z\left(t_{0}\right)=z_{0}, \quad t_{0} \in \mathcal{I},
\end{gathered}
$$

являющуюся специфической краевой задачей, в которой $\mathcal{L} *=\left(\mathcal{P}_{N(L)} *\right)\left(t_{0}\right)$.

Следствие 2. Пусть $L$ и $\mathcal{L}$ - обобщенно-обратимые операторы. Тогда задача Коши (11), (12) разрешима для тех и только тех $z_{0}=z\left(t_{0}\right) \in \mathbf{B}_{1} u \varphi(t) \in \mathbf{l}_{\infty}\left(\mathcal{I}, \mathbf{B}_{2}\right)$, которые удовлетворяют условиям

$$
\left(\mathcal{P}_{Y_{L}} \varphi\right)(t)=0, \quad \mathcal{P}_{Y_{\mathcal{L}}}\left\{z_{0}-\left(L^{-} \varphi\right)\left(t_{0}\right)\right\}=0
$$

и при этом имеет семейство решений

$$
z(t)=\left(\mathcal{P}_{N(L)} \mathcal{P}_{N(\mathcal{L})} \hat{z}\right)(t)+\left(G_{0} \varphi\right)(t)+\left(\mathcal{P}_{N(L)} \mathcal{L}^{-} z_{0}\right)(t)
$$

где $\mathcal{P}_{N(L)} \mathcal{P}_{N(\mathcal{L})}$ - разрешающий оператор соответствующей (11), (12) однородной $\left(z_{0}=0\right.$, $\varphi(t)=0)$ задачи Коши, $\hat{z}(t)$ - произвольный элемент банахова пространства $\mathbf{l}_{\infty}\left(\mathcal{I}, \mathbf{B}_{1}\right)$, $\left(G_{0} \varphi\right)(t)=\left(L^{-} \varphi\right)(t)-\left(\mathcal{P}_{N(L)} \mathcal{L}^{-}\left(L^{-} \varphi\right)\left(t_{0}\right)\right)(t)$ - оператор Грина полуоднородной $\left(z_{0}=0\right)$ задачи Коши (11), (12).

Замечание 3. Если $L$ - везде разрешимый дифференциальный оператор $(L z)(t)=$ $=z^{\prime}(t)-A(t) z(t)$, действующий из банахового пространства $\mathbf{C}^{1}(\mathcal{I}, \mathbf{B})$ непрерывно дифференцируемых функций со значениями в банаховом пространстве $\mathbf{B}$ в банахово пространство $\mathbf{C}(\mathcal{I}, \mathbf{B})$ непрерывных вектор-функций с супремум нормой и $\varphi(t)$ - непрерывная на промежутке $\mathcal{I}$ вектор-функция, то $\mathcal{P}_{Y_{L}} \equiv 0$, оператор $\mathcal{L} *=\left(\mathcal{P}_{N(L)} *\right)\left(t_{0}\right)$ обратимый для любого $t_{0} \in \mathcal{I}$, обобщенно-обратимый оператор $L^{-}$будет интегральным правым обратным оператором $L_{r}^{-1}$. В этом случае формула (13) примет вид [14, с. 148]

$$
z(t)=U\left(t, t_{0}\right) z_{0}+\int_{t_{0}}^{t} U(t, \tau) \varphi(\tau) d \tau, \quad t_{0} \in \mathcal{I},
$$

где $U(t, \tau)=U(t) U^{-1}(\tau)$ - эволюционный оператор.

Обобщенный оператор Грина и его свойства. Используя подход, предложенный в [32], краевую задачу (1), (2) запишем в векторно-матричном виде

$$
(\Lambda z)(t)=f(t)
$$

где $\Lambda=\operatorname{col}[L, \ell]$ - линейный оператор, $f(t)=\operatorname{col}[\varphi(t), \alpha] \in \mathbf{l}_{\infty}\left(\mathcal{I}, \mathbf{B}_{2}\right) \times \mathbf{B}$. 
Оператор $\Lambda: \mathbf{l}_{\infty}\left(\mathcal{I}, \mathbf{B}_{1}\right) \rightarrow \mathbf{l}_{\infty}\left(\mathcal{I}, \mathbf{B}_{2}\right) \times \mathbf{B}$ ограничен, если норму в пространстве $\mathbf{l}_{\infty}\left(\mathcal{I}, \mathbf{B}_{2}\right) \times \mathbf{B}$ ввести следующим образом:

$$
\|(\varphi, \alpha)\|_{\mathbf{l}_{\infty}\left(\mathcal{I}, \mathbf{B}_{2}\right) \times \mathbf{B}}=\|\varphi\|_{\mathbf{l}_{\infty}\left(\mathcal{I}, \mathbf{B}_{2}\right)}+\|\alpha\|_{\mathbf{B}}, \quad \varphi \in \mathbf{l}_{\infty}\left(\mathcal{I}, \mathbf{B}_{2}\right), \quad \alpha \in \mathbf{B} .
$$

Рассмотрим некоторые свойства обобщенного оператора Грина $(9) G: \mathbf{l}_{\infty}\left(\mathcal{I}, \mathbf{B}_{2}\right) \rightarrow \operatorname{ker} \ell \subset$ $\subset \mathbf{l}_{\infty}\left(\mathcal{I}, \mathbf{B}_{1}\right)$ и его связь с оператором $\Lambda$. Для упрощения записей в дальнейшем будем опускать скобки и переменные.

Лемма 1. Обобщенный оператор Грина (9) удовлетворяет соотношению

$$
\Lambda G=\left[\begin{array}{c}
I_{\mathbf{l}_{\infty}\left(\mathcal{I}, \mathbf{B}_{2}\right)}-\mathcal{P}_{Y_{L}} \\
\mathcal{P}_{Y_{\mathcal{L}}} \ell L^{-}
\end{array}\right]
$$

Доказательство. Действительно, подставив в (14) вместо $G$ его выражение (9), получим

$$
\begin{gathered}
\Lambda G=\left[\begin{array}{c}
L \\
\ell
\end{array}\right]\left[L^{-}-\mathcal{P}_{N(L)} \mathcal{L}^{-} \ell L^{-}\right]=\left[\begin{array}{c}
L\left(L^{-}-\mathcal{P}_{N(L)} \mathcal{L}^{-} \ell L^{-}\right) \\
\ell\left(L^{-}-\mathcal{P}_{N(L)} \mathcal{L}^{-} \ell L^{-}\right)
\end{array}\right]= \\
=\left[\begin{array}{c}
L L^{-}-L \mathcal{P}_{N(L)} \mathcal{L}^{-} \ell L^{-} \\
\ell L^{-}-\ell \mathcal{P}_{N(L)} \mathcal{L}^{-} \ell L^{-}
\end{array}\right]=\left[\begin{array}{c}
L L^{-} \\
\left(I_{\mathbf{B}}-\mathcal{L} \mathcal{L}^{-}\right) \ell L^{-}
\end{array}\right]=\left[\begin{array}{c}
I_{\infty}\left(\mathcal{I}, \mathbf{B}_{2}\right) \\
\mathcal{P}_{Y_{\mathcal{L}}} \ell L^{-}
\end{array}\right],
\end{gathered}
$$

так как $L \mathcal{P}_{N(L)}=0, \quad L L^{-}=I_{\mathbf{l}_{\infty}\left(\mathcal{I}, \mathbf{B}_{2}\right)}-\mathcal{P}_{Y_{L}}, \quad I_{\mathbf{B}}-\mathcal{L} \mathcal{L}^{-}=\mathcal{P}_{Y_{\mathcal{L}}}[11$, c. $53 ; 13$, c. 40].

Замечание 4 . В случае везде разрешимого уравнения $L z=\varphi$ соотношение (14) примет вид

$$
\Lambda G=\left[\begin{array}{c}
I_{\mathbf{l}_{\infty}\left(\mathcal{I}, \mathbf{B}_{2}\right)} \\
\mathcal{P}_{Y_{\mathcal{L}}} \ell L_{r}^{-1}
\end{array}\right]
$$

так как $R(L)=\mathbf{l}_{\infty}\left(\mathcal{I}, \mathbf{B}_{2}\right)$ и $\mathcal{P}_{Y_{L}} \equiv 0$, а $L^{-}=L_{r}^{-1}$.

Теорема 2. Пусть $L: \mathbf{l}_{\infty}\left(\mathcal{I}, \mathbf{B}_{1}\right) \rightarrow \mathbf{l}_{\infty}\left(\mathcal{I}, \mathbf{B}_{2}\right)$ и $\mathcal{L}: \mathbf{l}_{\infty}\left(\mathcal{I}, \mathbf{B}_{1}\right) \rightarrow \mathbf{B}$ - обобщенно обратимъие операторы. Тогда оператор

$$
\Lambda^{-}=\left[G, \mathcal{P}_{N(L)} \mathcal{L}^{-}\right]
$$

является ограниченным обобщенно-обратным $\kappa$ линейному ограниченному оператору $\Lambda=\operatorname{col}[L, \ell]$.

Доказательство. Как показано выше, при выполнении условий (3), (6) краевая задача

$$
\Lambda z=\left[\begin{array}{c}
L \\
\ell
\end{array}\right] z=\left[\begin{array}{l}
\varphi \\
\alpha
\end{array}\right]
$$

разрешима и ее решение имеет вид (10). Тогда для обобщенно-обратного оператора

$$
\Lambda^{-}: \mathbf{l}_{\infty}\left(\mathcal{I}, \mathbf{B}_{2}\right) \times \mathbf{B} \rightarrow \mathbf{l}_{\infty}\left(\mathcal{I}, \mathbf{B}_{1}\right)
$$

имеем представление

$$
\Lambda^{-} f=\left[\begin{array}{l}
L \\
\ell
\end{array}\right]^{-}\left[\begin{array}{l}
\varphi \\
\alpha
\end{array}\right]=G \varphi+\mathcal{P}_{N(L)} \mathcal{L}^{-} \alpha .
$$

Покажем, что оператор $\Lambda^{-}$обладает следующими свойствами:

1) $\Lambda^{-} \Lambda \Lambda^{-}=\Lambda^{-}$

2) $\Lambda \Lambda^{-} \Lambda=\Lambda$,

определяющими обобщенно-обратный оператор. Как отмечено в [24, с. 140], второе свойство является следствием первого. Поэтому для доказательства теоремы достаточно показать, что оператор $\Lambda^{-}$удовлетворяет равенству $\Lambda^{-} \Lambda \Lambda^{-}=\Lambda^{-}$. Так как $L \mathcal{P}_{N(L)} \equiv 0, \ell \mathcal{P}_{N(L)}=\mathcal{L}$, то

$$
\Lambda \Lambda^{-}=\left[\begin{array}{c}
L \\
\ell
\end{array}\right]\left[G, \mathcal{P}_{N(L)} \mathcal{L}^{-}\right]=\left[\begin{array}{cc}
L G & L \mathcal{P}_{N(L)} \mathcal{L}^{-} \\
\ell G & \ell \mathcal{P}_{N(L)} \mathcal{L}^{-}
\end{array}\right]=\left[\begin{array}{cc}
I_{\mathbf{l}_{\infty}\left(\mathcal{I}, \mathbf{B}_{2}\right)}-\mathcal{P}_{Y_{L}} & 0 \\
\mathcal{P}_{Y_{\mathcal{L}}} \ell L^{-} & \mathcal{L} \mathcal{L}^{-}
\end{array}\right]
$$


Из соотношений $L^{-} \mathcal{P}_{Y_{L}}=0, \mathcal{L}^{-} \mathcal{P}_{Y_{\mathcal{L}}}=0, G \mathcal{P}_{Y_{L}}=0$ следует, что

$$
\begin{gathered}
\Lambda^{-} \Lambda \Lambda^{-}=\left[G, \mathcal{P}_{N(L)} \mathcal{L}^{-}\right]\left[\begin{array}{cc}
I_{\mathbf{l}_{\infty}\left(\mathcal{I}, \mathbf{B}_{2}\right)}-\mathcal{P}_{Y_{L}} & 0 \\
\mathcal{P}_{Y_{\mathcal{L}}} \ell L^{-} & \mathcal{L} \mathcal{L}^{-}
\end{array}\right]= \\
=\left[G\left(I_{\mathbf{l}_{\infty}\left(\mathcal{I}, \mathbf{B}_{2}\right)}-\mathcal{P}_{Y_{L}}\right)+\mathcal{P}_{N(L)} \mathcal{L}^{-} \mathcal{P}_{Y_{\mathcal{L}}} \ell L^{-}, \mathcal{P}_{N(L)} \mathcal{L}^{-} \mathcal{L} \mathcal{L}^{-}\right]= \\
=\left[G-G \mathcal{P}_{Y_{L}}+\mathcal{P}_{N(L)} \mathcal{L}^{-} \mathcal{P}_{Y_{\mathcal{L}}} \ell L^{-}, \mathcal{P}_{N(L)} \mathcal{L}^{-}\right]=\left[G, \mathcal{P}_{N(L)} \mathcal{L}^{-}\right] .
\end{gathered}
$$

Ограниченность оператора $\Lambda^{-}$следует из ограниченности операторов $L^{-}, G$ и $\mathcal{P}_{N(L)} \mathcal{L}^{-}$. Таким образом, оператор $\Lambda^{-}: \mathbf{l}_{\infty}\left(\mathcal{I}, \mathbf{B}_{2}\right) \times \mathbf{B} \rightarrow \mathbf{l}_{\infty}\left(\mathcal{I}, \mathbf{B}_{1}\right)$ является ограниченным обобщеннообратным к оператору $\Lambda$ исходной краевой задачи.

Теорема 3. Условия разрешимости (3), (6) краевой задачи (1), (2) эквивалентны условию

$$
\mathcal{P}_{Y_{\Lambda}} f=0,
$$

где $\mathcal{P}_{Y_{\Lambda}}$ - проектор на нуль-пространство $N(\Lambda)$ оператора $\Lambda$.

Доказательство. Используя формулу [11, с. $53 ; 13$, с. 40$] \Lambda \Lambda^{-}=I_{\mathbf{l}_{\infty}\left(\mathcal{I}, \mathbf{B}_{2}\right) \times \mathbf{B}}-\mathcal{P}_{Y_{\Lambda}}$, связывающую обобщенно-обратный оператор $\Lambda^{-}$и проектор $\mathcal{P}_{Y_{\Lambda}}$, а также соотношение (16), найдем проектор $\mathcal{P}_{Y_{\Lambda}}$, который имеет блочную структуру:

$$
\begin{gathered}
\mathcal{P}_{Y_{\Lambda}}=I_{\mathbf{l}_{\infty}\left(\mathcal{I}, \mathbf{B}_{2}\right) \times \mathbf{B}}-\Lambda \Lambda^{-}=\left[\begin{array}{cc}
I_{\mathbf{l}_{\infty}\left(\mathcal{I}, \mathbf{B}_{2}\right)} & 0 \\
0 & I_{\mathbf{B}}
\end{array}\right]-\left[\begin{array}{cc}
I_{\mathbf{l}_{\infty}\left(\mathcal{I}, \mathbf{B}_{2}\right)}-\mathcal{P}_{Y_{L}} & 0 \\
\mathcal{P}_{Y_{\mathcal{L}}} \ell L^{-} & \mathcal{L} \mathcal{L}^{-}
\end{array}\right]= \\
=\left[\begin{array}{cc}
\mathcal{P}_{Y_{L}} & 0 \\
-\mathcal{P}_{Y_{\mathcal{L}}} \ell L^{-} & I_{\mathbf{B}}-\mathcal{L} \mathcal{L}^{-}
\end{array}\right]=\left[\begin{array}{cc}
\mathcal{P}_{Y_{L}} & 0 \\
-\mathcal{P}_{Y_{\mathcal{L}}} \ell L^{-} & \mathcal{P}_{Y_{\mathcal{L}}}
\end{array}\right] .
\end{gathered}
$$

Оператор $\mathcal{P}_{Y_{\Lambda}}$ действительно является проектором, так как обладает свойством $\mathcal{P}_{Y_{\Lambda}}^{2}=\mathcal{P}_{Y_{\Lambda}}$ :

$$
\begin{gathered}
\mathcal{P}_{Y_{\Lambda}}^{2}=\left[\begin{array}{cc}
\mathcal{P}_{Y_{L}} & 0 \\
-\mathcal{P}_{Y_{\mathcal{L}}} \ell L^{-} & \mathcal{P}_{Y_{\mathcal{L}}}
\end{array}\right]\left[\begin{array}{cc}
\mathcal{P}_{Y_{L}} & 0 \\
-\mathcal{P}_{Y_{\mathcal{L}}} \ell L^{-} & \mathcal{P}_{Y_{\mathcal{L}}}
\end{array}\right]= \\
=\left[\begin{array}{cc}
\mathcal{P}_{Y_{L}}^{2} & 0 \\
-\mathcal{P}_{Y_{\mathcal{L}}} \ell L^{-} \mathcal{P}_{Y_{L}}-\mathcal{P}_{Y_{\mathcal{L}}}^{2} \ell L^{-} & \mathcal{P}_{Y_{\mathcal{L}}}^{2}
\end{array}\right]=\left[\begin{array}{cc}
\mathcal{P}_{Y_{L}} & 0 \\
-\mathcal{P}_{Y_{\mathcal{L}}} \ell L^{-} & \mathcal{P}_{Y_{\mathcal{L}}}
\end{array}\right]=\mathcal{P}_{Y_{\Lambda}} .
\end{gathered}
$$

Таким образом, условие $\mathcal{P}_{Y_{\Lambda}} f=\left[\begin{array}{cc}\mathcal{P}_{Y_{L}} & 0 \\ -\mathcal{P}_{Y_{\mathcal{L}}} \ell L^{-} & \mathcal{P}_{Y_{\mathcal{L}}}\end{array}\right]\left[\begin{array}{c}\varphi \\ \alpha\end{array}\right]=0$ эквивалентно условиям разрешимости (3), (6) краевой задачи (1), (2). Теорема доказана.

Замечание 5. Если операторное уравнение (1) в краевой задаче (1), (2) является везде разрешимым, то проектор $\mathcal{P}_{Y_{\Lambda}}$ имеет вид

$$
\mathcal{P}_{Y_{\Lambda}}=\left[\begin{array}{cc}
0 & 0 \\
-\mathcal{P}_{Y_{\mathcal{L}}} \ell L_{r}^{-1} & \mathcal{P}_{Y_{\mathcal{L}}}
\end{array}\right]
$$

поскольку в этом случае $\mathcal{P}_{Y_{L}} \equiv 0$, а $L^{-}=L_{r}^{-1}$.

Пример. Рассмотрим операторное уравнение

$$
(L z)(t):=z(t)-M(t) \int_{0}^{1} N(s) z(s) d s=\varphi(t),
$$

где

$$
M(t)=\operatorname{diag}\left\{e^{t}, e^{t}, \ldots, e^{t}, e^{t}, \ldots\right\}, \quad N(s)=\operatorname{diag}\{s, 0, s, 0, \ldots, s, 0, \ldots\} .
$$


Пусть вектор-функция $\varphi(t)$ действует из отрезка $[0,1]$ в банахово пространство с всех сходящихся числовых последовательностей: $\varphi(t) \in \mathbf{C}([0,1], \mathbf{c}):=\{\varphi(\cdot):[0,1] \rightarrow \mathbf{c}\}$, операторфункции $M(t), N(t)$ действуют из банахова пространства $\mathbf{C}([0,1], \mathbf{c})$ в себя с нормами

$$
\|\mid M\|_{\mathbf{C}([0,1], \mathbf{c})}=\sup _{t \in[0,1]}\|M(t)\|_{\mathbf{c}}, \quad\|N\|_{\mathbf{C}([0,1], \mathbf{c})}=\sup _{t \in[0,1]}\|N(t)\|_{\mathbf{c}} .
$$

Из определения оператор-функций $M(t)$ и $N(t)$ следует, что

$$
\||| M||_{\mathbf{C}([0,1], \mathbf{c})}=\sup _{\substack{t \in[0,1] \\ i, j \in \mathbb{N}}}\left|m_{i j}(t)\right|=\sup _{t \in[0,1]}\left|e^{t}\right| \leq e, \quad|||N||| \mathbf{C}([0,1], \mathbf{c})=\sup _{\substack{t \in[0,1] \\ i, j \in \mathbb{N}}}\left|n_{i j}(t)\right|=\sup _{\substack{t \in[0,1] \\ \mid}}|t| \leq 1 .
$$

Тогда

$$
\begin{gathered}
\|L\|_{\mathbf{C}([0,1], \mathbf{c})}=\sup _{\substack{z \in \mathbf{C}([0,1], \mathbf{c}) \\
z \neq 0}} \frac{\|L z\|_{\mathbf{C}([0,1], \mathbf{c})}}{\|z\|_{\mathbf{C}([0,1], \mathbf{c})}}=\sup _{\substack{z \in \mathbf{C}([0,1], \mathbf{c}) \\
z \neq 0}} \frac{\left\|z(t)+M(t) \int_{0}^{1} N(s) z(s) d s\right\|_{\mathbf{C}([0,1], \mathbf{c})}}{\|z\|_{\mathbf{C}([0,1], \mathbf{c})}} \leq \\
\leq \sup _{z \in \mathbf{C}([0,1], \mathbf{c})} \frac{\|z(t)\|_{\mathbf{C}([0,1], \mathbf{c})}+\|M(t)\|_{\mathbf{C}([0,1], \mathbf{c})} \int_{0}^{1}\|N(s)\|_{\mathbf{C}([0,1], \mathbf{c})}\|z(s)\|_{\mathbf{C}([0,1], \mathbf{c})} d s}{\|z\|_{\mathbf{C}([0,1], \mathbf{c})}} \leq \\
\leq \sup _{\substack{z \in \mathbf{C}([0,1], \mathbf{c}) \\
z \neq 0}} \frac{(1+e)\|z(t)\|_{\mathbf{C}([0,1], \mathbf{c})}}{\|z\|_{\mathbf{C}([0,1], \mathbf{c})}} \leq 1+e .
\end{gathered}
$$

Таким образом, оператор $L$ является линейным ограниченным оператором, действующим из банахового пространства непрерывных на промежутке $[0,1]$ функций $\mathbf{C}([0,1], \mathbf{c})$ в себя.

Найдем решения уравнения (17), удовлетворяющие условиям

$$
\ell z(\cdot)=\int_{0}^{1} S(t) z(t) d t=\alpha,
$$

где

$$
\begin{gathered}
S(t)=\operatorname{diag}\left\{S_{(2 \times 4)}(t), S_{(2 \times 4)}(t), \ldots\right\}, \quad S_{(2 \times 4)}(t)=\left(\begin{array}{cccc}
e^{-t} & 0 & e^{-t} & 0 \\
(2 t-1) e^{-t} & 0 & 0 & 0
\end{array}\right), \\
\|S \mid\|_{\mathbf{C}([0,1], \mathbf{c})}=\sup _{t \in[0,1]}\|S(t)\|_{\mathbf{c} .}
\end{gathered}
$$

Вектор-функционал $\ell$ действует из пространства $\mathbf{C}([0,1], \mathbf{c})$ в банахово пространство с и является ограниченным, $\alpha \in \mathbf{c}, \alpha=\operatorname{col}\left(\alpha_{1}, \alpha_{2}, \alpha_{3}, \ldots\right)$.

Для оператора $L$ ограниченные проекторы $\mathcal{P}_{N(L)}, \mathcal{P}_{Y_{L}}$ и операторы $\overline{\mathcal{P}}_{Y_{L}}, \overline{\mathcal{P}}_{N(L)}$ примут соответственно вид

$$
\begin{aligned}
& \left(\mathcal{P}_{N(L)} z\right)(t)=X(t) \int_{0}^{1} \Gamma(s) z(s) d s, \quad\left(\mathcal{P}_{Y_{L}} y\right)(t)=\Psi \int_{0}^{1} \Phi(s) y(s) d s \\
& \left(\overline{\mathcal{P}}_{Y_{L}} z\right)(t)=\Psi \int_{0}^{1} \Gamma(s) z(s) d s, \quad\left(\overline{\mathcal{P}}_{N(L)} y\right)(t)=X(t) \int_{0}^{1} \Phi(s) y(s) d s,
\end{aligned}
$$

ДИФФЕРЕНЦИАЛЬНЫЕ УРАВНЕНИЯ 
где

$$
\begin{aligned}
& X(t)=\operatorname{diag}\left\{X_{(4 \times 2)}(t), X_{(4 \times 2)}(t), \ldots\right\}, \quad \Gamma(t)=\operatorname{diag}\left\{\Gamma_{(2 \times 4)}(t), \Gamma_{(2 \times 4)}(t), \ldots\right\}, \\
& X_{(4 \times 2)}(t)=\left(\begin{array}{cccc}
e^{t} & 0 & 0 & 0 \\
0 & 0 & e^{t} & 0
\end{array}\right)^{\mathrm{T}}, \quad \Gamma_{(2 \times 4)}(t)=\left(\begin{array}{cccc}
t & 0 & 0 & 0 \\
0 & 0 & t & 0
\end{array}\right), \\
& \Phi(t)=\operatorname{diag}\left\{\Phi_{(2 \times 4)}(t), \Phi_{(2 \times 4)}(t), \ldots\right\}, \quad \Psi(t)=\operatorname{diag}\left\{\Psi_{(4 \times 2)}(t), \Psi_{(4 \times 2)}(t), \ldots\right\}, \\
& \Phi_{(2 \times 4)}(t)=\left(\begin{array}{cccc}
t & 0 & 0 & 0 \\
0 & 0 & t & 0
\end{array}\right), \quad \Psi_{(4 \times 2)}(t)=\left(\begin{array}{llll}
2 & 0 & 0 & 0 \\
0 & 0 & 2 & 0
\end{array}\right)^{\mathrm{T}} .
\end{aligned}
$$

Из ограниченности проекторов $\mathcal{P}_{N(L)}$ и $\mathcal{P}_{Y_{L}}$ следует обобщенная обратимость, а следовательно, нормальная разрешимость оператора $L$. Поэтому исходное уравнение $(17)$ будет разрешимо тогда и только тогда, когда вектор-функция $\varphi(t)$ удовлетворяет условию

$$
\left(\mathcal{P}_{Y_{L}} \varphi\right)(t)=\Psi \int_{0}^{1} \Phi(s) \varphi(s) d s=0
$$

которое будет выполнено тогда и только тогда, когда компоненты вектор-функции $\varphi(t)=$ $=\operatorname{col}\left(\varphi_{1}(t), \varphi_{2}(t), \varphi_{3}(t), \ldots,\right)$ будут удовлетворять соотношениям

$$
\int_{0}^{1} s \varphi_{2 k-1}(s) d s=0, \quad k=1,2,3, \ldots
$$

При выполнении условий (19) операторное уравнение (17) будет иметь решение [11, с. 82; 13 , с. $69 ; 31]$

$$
z(t)=\left(\mathcal{P}_{N(L)} \hat{z}\right)(t)+\left(L^{-} \varphi\right)(t)=X(t) \int_{0}^{1} \Gamma(s) \hat{z}(s) d s+\varphi(t)+M_{1}(t) \int_{0}^{1} N_{1}(s) \varphi(s) d s
$$

где $\hat{z}(t)-$ произвольный элемент пространства $\mathbf{C}([0,1], \mathbf{c})$,

$$
\begin{gathered}
M_{1}(t)=\operatorname{diag}\left\{M_{(2 \times 4)}(t), M_{(2 \times 4)}(t), \ldots\right\}, \quad N_{1}(s)=\operatorname{diag}\left\{N_{(4 \times 2)}(s), N_{(4 \times 2)}(s), \ldots\right\}, \\
M_{(2 \times 4)}(t)=\left(\begin{array}{cccc}
2\left(e^{t}-1\right) & 0 & -e^{t} & -e^{t} \\
0 & e^{t} & 0 & 0
\end{array}\right), \quad N_{(4 \times 2)}(s)=\left(\begin{array}{cccc}
s & 0 & s & s \\
0 & 0 & 0 & 0
\end{array}\right)^{\mathrm{T}} .
\end{gathered}
$$

Подставляя общее решение (20) в краевое условие (18), получаем операторное уравнение

$$
\mathcal{L} \hat{z}+\ell\left(L^{-} \varphi\right)(\cdot)=\alpha
$$

где

$$
(\mathcal{L} \hat{z})(\cdot)=\left(\ell \mathcal{P}_{N(L)} \hat{z}\right)(\cdot)=\int_{0}^{1} S(t) X(t) \int_{0}^{1} \Gamma(s) \hat{z}(s) d s d t=Q \int_{0}^{1} \Gamma(s) \hat{z}(s) d s .
$$

Линейный ограниченный матричный оператор

$$
Q=\int_{0}^{1} S(t) X(t) d t=\operatorname{diag}\left\{\left(\begin{array}{ll}
1 & 1 \\
0 & 0
\end{array}\right),\left(\begin{array}{ll}
1 & 1 \\
0 & 0
\end{array}\right), \ldots\right\}
$$

действует из банахова пространства с ограниченных, сходящихся к конечному пределу последовательностей в себя, $Q: \mathbf{c} \rightarrow \mathbf{c}$ и является обобщенно обратимым. 
Обозначив $\hat{z}_{0}=\int_{0}^{1} \Gamma(s) \hat{z}(s) d s$, запишем операторное уравнение $(21)$ в виде матричного операторного уравнения

$$
Q \hat{z}_{0}=\alpha-\ell \varphi(\cdot)-\ell M_{1}(\cdot) \int_{0}^{1} N_{1}(s) \varphi(s) d s .
$$

Построив проектор $\mathcal{P}_{Y_{Q}}: \mathbf{c} \rightarrow Y_{Q}:$

$$
\mathcal{P}_{Y_{Q}}=\operatorname{diag}\left\{\left(\begin{array}{ll}
0 & 0 \\
0 & 1
\end{array}\right),\left(\begin{array}{ll}
0 & 0 \\
0 & 1
\end{array}\right), \ldots\right\}
$$

получим, что для разрешимости уравнения (22) необходимо и достаточно, чтобы выполнялось условие

$$
\mathcal{P}_{Y_{Q}}\left\{\alpha-\ell \varphi(\cdot)-\ell M_{1}(\cdot) \int_{0}^{1} N_{1}(s) \varphi(s) d s\right\}=0 .
$$

После преобразований получим, что компоненты вектора $\alpha \in \mathbf{c}$ и вектор-функции $\varphi(t) \in$ $\in \mathbf{C}([0,1], \mathbf{c})$ должны удовлетворять условиям

$$
\alpha_{2 k}-\int_{0}^{1}(2 s-1) e^{-s} \varphi_{4 k-3}(s) d s-\frac{6-2 e}{e} \int_{0}^{1} s \varphi_{8 k-7}(s) d s=0, \quad k=1,2,3, \ldots
$$

При выполнении этих условий операторное уравнение (22) будет иметь решение

$$
\hat{z}_{0}=\mathcal{P}_{N(Q)} c+Q^{-}\left\{\alpha-\ell \varphi(\cdot)-\ell M_{1}(\cdot) \int_{0}^{1} N_{1}(s) \varphi(s) d s\right\},
$$

где $c$ - произвольный элемент пространства с

$$
\mathcal{P}_{N(Q)}=\operatorname{diag}\left\{\left(\begin{array}{cc}
1 & 0 \\
-1 & 0
\end{array}\right),\left(\begin{array}{cc}
1 & 0 \\
-1 & 0
\end{array}\right), \ldots\right\}, \quad Q^{-}=\operatorname{diag}\left\{\left(\begin{array}{ll}
0 & 0 \\
1 & 0
\end{array}\right),\left(\begin{array}{ll}
0 & 0 \\
1 & 0
\end{array}\right), \ldots\right\} .
$$

Подставляя найденное $\hat{z}_{0}$ из (24) вместо $\int_{0}^{1} \Gamma(s) \hat{z}(s) d s$ в (20), получаем общее решение краевой задачи $(17),(18)$

$$
\begin{gathered}
z(t)=X(t) \mathcal{P}_{N(Q)} c+\varphi(t)-X(t) Q^{-} \ell \varphi(\cdot)- \\
-X(t) Q^{-} \ell M_{1}(\cdot) \int_{0}^{1} N_{1}(s) \varphi(s) d s+M_{1}(t) \int_{0}^{1} N_{1}(s) \varphi(s) d s+X(t) Q^{-} \alpha .
\end{gathered}
$$

Таким образом, краевая задача (17), (18) разрешима тогда и только тогда, когда выполнены условия (19), (23), и в этом случае она будет иметь семейство решений

$$
\begin{gathered}
z(t)=X(t) \mathcal{P}_{N(Q)} c+\left[\varphi(t)-X(t) Q^{-} \ell \varphi(\cdot)\right]+ \\
+\left[M_{1}(t)-X(t) Q^{-} \ell M_{1}(\cdot)\right] \int_{0}^{1} N_{1}(s) \varphi(s) d s+X(t) Q^{-} \alpha .
\end{gathered}
$$

\section{СПИСОК ЛИТЕРАТУРЫ}

1. Ляпунов A.М. Общая задача об устойчивости движения. М., 1950.

2. Малкин И.Г. Некоторые задачи теории нелинейных колебаний. М., 1956.

3. Гребеников Е.А., Рябов Ю.А. Конструктивные методы анализа нелинейных систем. М., 1979. 
4. Хейл Дж.К. Теория функционально-дифференциальных уравнений. М., 1984.

5. Самойленко А.М., Перестюк Н.А. Дифференциальные уравнения с импульсным воздействием. Киев, 1987.

6. Tvrdy M., Veivoda $O$. General boundary value problems for an integrodifferential system and its adjoint // C̆as. prestov. mat. 1973. V. 98. P. 26-42.

7. Schwabic S̆., Tvrdy M., Veivoda O. Differential and integral equations. Boundary value problems and adjoints. Praga, 1979.

8. Mawhin J. Topological degree methods in nonlinear boundary value problems: Regional conference series in mathematics. Providence, 1979. № 40.

9. Ben-Israel A., Greville T.N.E. Generalized Inverse Theory and Applications (second edition). New York, 2003.

10. Бойчук А.А. Конструктивные методы анализа краевых задач. Киев, 1990.

11. Бойчук А.А., ЖУравлев В.Ф., Самойленко А.М. Обобщенно-обратные операторы и нётеровы краевые задачи. Киев, 1995.

12. Бойчук А.А., Журавлев В.Ф., Самойленко А.М. Линейные нётеровы краевые задачи для импульсных дифференциальных систем с запаздыванием // Дифференц. уравнения. 1994. Т. 30. № 10. C. $1677-1682$.

13. Boichuk A.A., Samoilenko A.M. Generalised inverse operators and Fredholm boundary-value problems. Utrecht; Boston: VSP, 2004.

14. Далецкий Ю.Л., Крейн М.Г. Устойчивость решений дифференциальных уравнений в банаховом пространстве. М., 1970.

15. Бойчук О.А., Панасенко Э.В. Крайові задачі для диференціальних рівнянь у банаховому просторі // Нелінійні коливання. 2009. Т. 12. № 1. С. 16-19.

16. Самойленко А.М., Теплінсъкий Ю.В. Елементи математичної теорії еволюційних рівнянь у банахових просторах. Киев, 2008.

17. Boichuk A., Pokutnij A. Bounded solutions of linear perturbed differential equations in a Banach space // Tatra Mt. Math. Publ. 2007. V. 39. P. 1-12.

18. Крейн С.Г. Линейные уравнения в банаховом пространстве. М., 1971.

19. Samoilenko A.M., Boichuk A.A., Krivosheya S.A. Boundary-value problem for linear systems of integrodifferential equations with degenerate kernel // Ukr. Mat. Zh. 1996. V. 48. № 11. P. 1576-1579.

20. Samoilenko A.M., Shkil' M.I., Yakovec' V.P. Linear systems of differential equations with Singularities. Kyiv, 2000.

21. Чистлков В.Ф., Щеглова А.А. Избранные главы теории алгебро-дифференциальных систем. Новосибирск, 2003.

22. Boichuk A.A., Shegda L.M. Bifurcation of Solutions of Singular Fredholm Boundary Value Problems // Differ. Equat. 2011. V. 47. № 4. P. 453-461.

23. Хелемский А.Я. Лекции по функциональному анализу. М., 2004.

24. Гохберг И.Ц., Крупник Н.Я. Введение в теорию одномерных сингулярных интегральных операторов. Кишинев, 1973.

25. Попов М.М. Доповнювальні простори і деякі задачі сучасної геометрії просторів Банаха // Математика сьогодні ’07. 2007. Вип. 13. С. 78-116.

26. Fredholm I. Sur une Classe d'equations fonctionnelles // Acta Math. 1903. V. 27. P. 265-390.

27. Noether F. Über eine Klasse singulä Integralgleichungen // Math. Ann. 1921. № 82. P. 42-64.

28. Королюк В.С., Турбин А.Ф. Математические основы фазового укрупнения сложных систем. Киев, 1978.

29. Абдуллаев А.Р., Бурмистрова А.Б. Топологические нётеровы операторы: обобщенная обратимость и аддитивное представление // Изв. вузов. 1994. № 6. С. 3-7.

30. Generalized Inverses and Applications / Ed. by Nashed M.Z. New York; San Francisco; London. 1976.

31. Журавлев В.Ф. Критерий разрешимости и представление решений линейных $n-(d-)$ нормальных операторных уравнений в банаховом пространстве // Укр. мат. журн. 2010. Т. 62. № 2. С. 167-182.

32. Wexler D. On Boundary Value Problems for an Ordinary Linear Differential Systems // Ann. di Mat. pura et Appl. 1968. V. 80. P. 122-136.

Институт математики НАН Украины,

Поступила в редакцию

г. Киев, 31.12 .2012 г.

Житомирский национальный

агроэкологический университет 